\title{
Non-circular rotating beams and CMB experiments
}

\author{
J. V. Arnau ${ }^{1}$, A. M. Aliaga ${ }^{2}$, and D. Sáez ${ }^{2}$ \\ 1 Departamento de Matemática Aplicada, Universidad de Valencia, 46100 Burjassot, Valencia, Spain \\ e-mail: jose.arnau@uv.es \\ 2 Departamento de Astronomía y Astrofísica, Universidad de Valencia, 46100 Burjassot, Valencia, Spain
}

Received 10 August 2001 / Accepted 22 November 2001

\begin{abstract}
This paper is concerned with small angular scale experiments for the observation of cosmic microwave background anisotropies. In the absence of beam, the effects of partial coverage and pixelisation are disentangled and analyzed (using simulations). Then, appropriate maps involving the CMB signal plus the synchrotron and dust emissions from the Milky Way are simulated, and an asymmetric beam - which turns following different strategies - is used to smooth the simulated maps. An associated circular beam is defined to estimate the deviations in the angular power spectrum produced by beam asymmetry without rotation and, afterwards, the deviations due to beam rotation are calculated. For a certain large coverage, the deviations due to pure asymmetry and asymmetry plus rotation appear to be very systematic (very similar in each simulation). Possible applications of the main results of this paper to data analysis in large coverage experiments - as PLANCK - are outlined.
\end{abstract}

Key words. cosmology: cosmic microwave background - cosmology: theory - large-scale structure of the universe methods: numerical - methods: data analysis

\section{Introduction}

Many experiments are being designed for the observation of the Cosmic Microwave Background (CMB) anisotropies. From the maps of a given experiment operating with a non-circular (asymmetric) rotating beam, a certain angular power spectrum $\left(C_{\ell}\right.$ quantities) can be extracted. Different rotations can lead to distinct $C_{\ell}$ coefficients and, the question is: how different are these coefficients? In other words, how relevant is the effect of the rotation strategy on the resulting angular power spectrum?

In a previous paper (Arnau \& Sáez 2000), it was shown that, in the absence of rotation and when the level of instrumental noise is low enough, the effect of a non-circular beam can be subtracted - namely, the beam can be deconvolved - using the Fourier transform. This subtraction can be performed in such a way that the resulting spectrum, after deconvolution, is very similar to the true one. That is possible if the number of pixels inside the beam, $N_{\text {in }}$, is not too great. Indeed, $N_{\text {in }}$ cannot be much greater than 10; however, if the beam rotates, the deconvolution is not possible. Nobody has described either the importance of beam rotation or a method to eliminate its effects. The main goal of this paper is the estimation of the effects due to rotation. In Arnau \& Sáez 2000 (and also in Sáez et al. 1996; Sáez \& Arnau 1997), a sort of modified angular

Send offprint requests to: D. Sáez, e-mail: diego.saez@uv.es power spectrum was used. Here, we extract the standard $C_{\ell}$ quantities from a certain number of squared patches of the sky. Recently, Wu et al. (2001) have proposed a method for data analysis in the case of asymmetric beams. This method is based on an optimal circular beam associated to the asymmetric one. The effects of beam rotation are not studied at all by these authors.

Although our methods apply to CMB anisotropy experiments in general, we will pay particular attention to PLANCK mission (scheduled by ESA for 2007). As it was emphasized in Burigana et al. (1998), beam responses are typically nonsymmetric for detectors de-centred from the telescope focus. Taking into account that CMB anisotropy experiments require observations at different frequencies, various detectors are necessary, which must be distributed as close as possible from the focus; for instance, in the PLANCK mission, around one hundred of detector (bolometers and radiometers) must be distributed in the focal plane. If the focal plane rotates (rotation of the telescope around the spin axis), the beams do. The effect of this rotation deserves attention. Furthermore, there are various identical detectors for each frequency, which are located at different positions in the focal plane and, consequently, the deformations of these beams would be different (identical) if they are located at different (the same) distances from the optical focus; nevertheless, even for identical deformations, the orientations of the 
resulting asymmetric beams would be different. The motion of the line of sight through the sky also produces a beam asymmetry. The effective beam diameter $\theta_{\text {Fwнм }}$ appears to be enhanced in the direction of this motion (see Hanany et al. 1998). This small effect is due to the beam displacement during the measurement process. It is not taken into account in this paper.

Beam rotation depends on the particular experiment under consideration. Given a pixelisation, the beam centre points towards a given pixel a certain number of times, $N_{\mathrm{p}}$, and, then, the temperature assigned to this pixel is an average of the temperatures corresponding to each of the $N_{\mathrm{p}}$ measurements. The fact that measurements from various beam orientations are averaged could be important.

In the case of PLANCK mission, a rough estimate of number $N_{\mathrm{p}}$ is given in Sáez \& Arnau (2000). Here, it is worthwhile to improve a little on that estimate. The satellite has been designed in such a way that: (i) it will cover the full sky in seven months, with a coverage which can be considered as uniform in most part of the sky, (ii) its line of sight will move around a big circle on the sky completing a turn each minute and, (iii) it will move around the same circle for two hours (120 turns). On account of these facts, if the pixel size is $\Delta$ and the angle subtended by the motion of the line of sight between two successive measurements is $\Delta \alpha=\zeta \theta_{\text {FWнM }}$, where $\theta_{\text {FWнм }}$ is the beam diameter, then, the average number of measurements per pixel (in a seven months observing period) is $N_{\mathrm{p}}=42 \Delta^{2} / \zeta \theta_{\text {FWHM }}$, where all the angles are given in arc-minutes (see Sáez \& Arnau 2000, for comparison) and, furthermore, the average number of measurements per pixel performed while the line of sight turns 120 times around a given circle is $N_{\mathrm{pc}}=120 \Delta / \zeta \theta_{\mathrm{FWHM}}$. From these formulae, it follows that the average number of circles passing by a pixel - during seven months - is $N_{\mathrm{c}}=N_{\mathrm{p}} / N_{\mathrm{pc}} \simeq \Delta / 3$, this result is consistent with the fact that, for a given observational strategy, the number $N_{\mathrm{c}}$ is expected to be dependent only on the pixelisation. Of course, it is independent on beam asymmetry. The number of measurements corresponding to different orientations could be important for the effect we are looking for, which is produced by the rotation of asymmetric beams. The larger the pixel size, the better the situation (the greater $N_{\mathrm{c}}$ ).

Since the detectors are rigidly attached to the focal plane, any beam has almost the same orientation each time it crosses a given pixel during its motion (120 turns) along a given circle; however, this orientation changes from circle to circle. From the above comments and estimates, it follows that the average number of measurements per pixel corresponding to different beam orientations is $N_{\mathrm{c}}$. If the full sky is covered two times and, the second coverage is not identical to the first one, this average number would be $2 \Delta / 3$. For $5^{\prime}<\Delta<10^{\prime}$, this number ranges from 3.3 to 6.6 . Nevertheless, there are various detectors in the focal plane for each frequency and, by assuming that all the beams have the same shape but different orientations, the above $N_{\mathrm{c}}$ number can be multiplied by the number of beams.

\section{Beam}

Our asymmetric beam is assumed to be of the form:

$W(\theta, \phi)=W_{\mathrm{N}} \mathrm{e}^{\left[-\frac{\left(\theta-\theta^{\prime}\right)^{2}}{2 \sigma_{\theta}^{2}}-\frac{\left(\phi-\phi^{\prime}\right)^{2}}{2 \sigma_{\phi}^{2}}\right]}$

as in Burigana et al. (1998). It is hereafter called an elliptical beam. Estimations of the effects due to asymmetry require the definition of an associated circularly symmetric beam for comparisons. Here the associated beam is defined as follows: given a circle of radius $R=\left(\theta^{2}+\phi^{2}\right)^{1 / 2}$, the weight $W(R)$ corresponding to the circular beam is the average value of the weights $W(\theta, \phi)$ assigned by the elliptical beam to the points of the circle; namely,

$W(R)=\langle W(\theta, \phi)\rangle_{\mathrm{C}}$.

There are arguments suggesting that the circular beam given by Eq. (2) is very appropriate. Let us discuss this point in some detail. Suppose an uniform temperature field $T(\theta, \phi)=$ constant. Place the centre of the elliptical beam at point $\mathrm{P}$ with arbitrary orientation and, then, consider various circles centred at $\mathrm{P}$ with radius $R_{i}$. The averages inside each of these circles performed with the asymmetrical beam and with the associated circular one are identical (except for errors produced by discretisation in Eqs. (1) and (2)). These averages coincide whatever the radius $R_{i}$ and the orientation of the asymmetric beam may be. With any other circular beam, there would be deviations between the measurements of both beams, which would depend on the spatial scale (on $R_{i}$ ), that feature does not seem to be appropriate because scale dependent differences would appear as an artifact. It could be argued that we are considering a very special uniform field which is very different from the true CMB maps; nevertheless, in spite of the fact that the cosmological signal has fluctuations at different scales, this signal is expected to be an homogeneous and isotropic statistical field and, consequently, the above associated beams seem to be appropriate in order to avoid artifacts after measurements and averages; namely, from a statistical point of view. The differences between the angular power spectra after averaging with the two associated beams are not artifacts due to a bad association, but small differences produced by other numerical or physical reasons. For example, as a result of pixelisation (a type of discretisation) beams (1) and (2) deviate. It is worthwhile to notice that the associated circular beam can be easily obtained - using Eq. (2) whatever the asymmetric beam may be and, also, that this association does not depend on the features of the maps to be smoothed by the beam (which are not known a priori). In the case of the beam defined by Eq. (1), the associated circular beam appears to have the following form:

$W(R)=\frac{2 W_{\mathrm{N}}}{\pi} \mathrm{e}^{-R^{2} \sigma_{\phi}^{-2} / 2} \int_{0}^{\pi / 2} \mathrm{e}^{\left[R^{2}\left(\sigma_{\theta}^{-2}-\sigma_{\phi}^{-2}\right) \sin ^{2} \xi\right] / 2} \mathrm{~d} \xi$,

which is used below in numerical estimates.

The total signal - measured in a certain frequency also involves components which are not statistically homogeneous and isotropic; for instance, dust and synchrotron 
radiations from our galaxy; nevertheless, we cannot use two different circular associated beams, but only one, and the fact that we are particularly interested in the CMB signal strongly suggests the use of beam (2).

\section{Map making algorithm and power spectrum estimator}

We are concerned with a $\Lambda C D M$ model, which is a standard inflationary (flat) one with cold dark matter, having $\Omega_{\mathrm{b}}=0.05, \Omega_{\mathrm{d}}=0.25, \Omega_{\Lambda}=0.7$, and $h=0.65$, where quantities $\Omega_{\mathrm{b}}, \Omega_{\mathrm{d}}$ and $\Omega_{\Lambda}$ stand for the density parameters corresponding to baryons, dark matter, and vacuum energy densities, respectively, and quantity $\mathrm{h}$ is the reduced Hubble constant. In this model, the CMB temperature is a Gaussian homogeneous and isotropic statistical two dimensional field. In such a case, a certain method proposed by Bond \& Efstathiou (1987) can be used to make the $18^{\circ} \times 18^{\circ}$ maps used in this paper. This method is based on the following formula:

$$
\frac{\delta T}{T}=\sum_{s_{1}, s_{2}=-N}^{N} D\left(\ell_{1}, \ell_{2}\right) \mathrm{e}^{-i\left(\theta \ell_{1}+\phi \ell_{2}\right)},
$$

where $\ell_{1}=2 \pi s_{1} / \Lambda, \ell_{2}=2 \pi s_{2} / \Lambda$, and $\Lambda$ stands for the angular size of the square to be mapped. This equation defines a Fourier transform from the position space $(\theta, \phi)$ to the momentum space $\left(\ell_{1}, \ell_{2}\right)$. The Gaussian quantities $D\left(\ell_{1}, \ell_{2}\right)$ have zero mean, and their variance is proportional to $C_{\ell}$, where $\ell=\left(\ell_{1}^{2}+\ell_{2}^{2}\right)^{1 / 2}$. Since $\delta T / T$ is real, the relation $D\left(-\ell_{1},-\ell_{2}\right)=D^{*}\left(\ell_{1}, \ell_{2}\right)$ must be satisfied. From given $C_{\ell}$ coefficients, the above $D\left(\ell_{1}, \ell_{2}\right)$ quantities can be easily calculated and, then, according to Eq. (4), a Fourier transform leads to the map. Sáez et al. (1996) used this map making algorithm to get very good simulations of $20^{\circ} \times 20^{\circ}$ squared regions.

In the case of small squared maps, the above map making method suggests a power spectrum estimator. Given one of these maps $\delta T / T(\theta, \phi)$, an inverse Fourier transform leads to quantities $D\left(\ell_{1}, \ell_{2}\right)$ and, then, the average $\left\langle\left|D\left(\ell_{1}, \ell_{2}\right)\right|^{2}\right\rangle$ can be calculated on the circumference $\ell^{2}=\ell_{1}^{2}+\ell_{2}^{2}$. Some interpolations are necessary to get the $D\left(\ell_{1}, \ell_{2}\right)$ values at the points located on the circumference. The resulting average is proportional to $C_{\ell}$, where $\ell$ is the radius of the circumference.

Another map making algorithm and a different power spectrum estimator have been also used for comparisons. A few comments about these methods, which play an auxiliary role in this paper, are worthwhile.

The effect of partial coverage - without considering pixelisation - was studied by Scott et al. (1994). In an experiment covering a fraction, $f_{\text {sky }}$, of the sky, these authors showed that the sample variance is just the cosmic one enhanced by the factor $f_{\text {sky }}^{-1}$. The meaning of the cosmic variance was discussed in detail by L. Nox (1995). From these papers it follows that, in an experiment with partial coverage, the deviations of the estimated angular power spectrum $C_{\ell}^{\text {est }}$ with respect to the average $C_{\ell}=\left\langle C_{\ell}^{\text {est }}\right\rangle$ obey the relation $\left\langle\left(C_{\ell}^{\mathrm{est}}-C_{\ell}\right)\left(C_{\ell^{\prime}}^{\mathrm{est}}-C_{\ell^{\prime}}\right)\right\rangle=\left[2 /(2 \ell+1) f_{\mathrm{sky}}\right] C_{\ell}^{2} \delta_{\ell \ell^{\prime}}$ and, consequently, the relative errors of the resulting $C_{\ell}$ quantities are:

$$
\frac{\Delta C_{\ell}}{C_{\ell}}=\left[\frac{2}{(2 \ell+1) f_{\text {sky }}}\right]^{1 / 2} \text {. }
$$

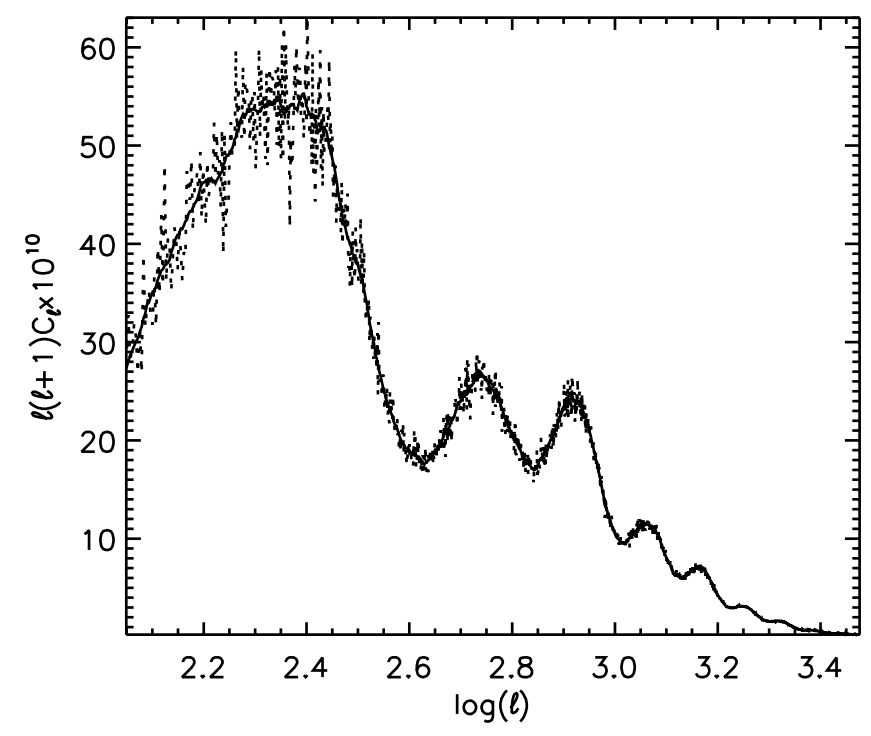

Fig. 1. Dotted line shows the angular power spectrum $C_{\ell}^{\text {est }}$, which involves high frequency oscillations (sample variance), whereas solid line approaches the true spectrum $C_{\ell}=\left\langle C_{\ell}^{\text {est }}\right\rangle$.

In Fig. 1, two angular power spectra are displayed. One of them (dotted line) has been directly calculated using the HEALPIX (Hierarchical Equal Area isoLatitude Pizelisation) package. First, a full sky simulation has been made using the code SYNFAST with the following inputs: (1) the $C_{\ell}$ coefficients of the $\Lambda C D M$ model under consideration, and (2) a pixel size $\Delta=3.435\left(N_{\text {side }}=1024\right)$ and, then, the resulting map has been analyzed using the code ANAFAST, which gives the angular power spectrum. We have excluded an equatorial band in the analysis and, consequently, the resulting spectrum corresponds to two polar regions covering a part of the sky with $f_{\text {sky }} \simeq 0.39$. Since ANAFAST gives the quantities $C_{\ell}^{\text {est }}$ defined above, the dotted line shows high frequency oscillations (sample variance). We can now use some appropriate numerical method to estimate the quantities $C_{\ell}=\left\langle C_{\ell}^{\text {est }}\right\rangle$. A simple method suffices for us, we have taken $\left\langle C_{\ell}^{\text {est }}\right\rangle=\frac{1}{21} \sum_{i=\ell-10}^{\ell+10} C_{i}^{\text {est }}$. The resulting smooth spectrum is that of the solid line. The high frequency oscillations due to partial coverage have been partially subtracted from the spectrum displayed in the dotted line; thus, the solid line shows a spectrum which only involves a residual part of the effect due to partial coverage. This residual effect should be also proportional to $f_{\text {sky }}^{-1}$.

The power spectrum estimator based on the Fourier transform directly approaches quantities $C_{\ell}=\left\langle C_{\ell}^{\text {est }}\right\rangle$; namely, the spectrum given by this estimator does not involve high frequency oscillations, but only a residual 
effect of partial coverage proportional to $f_{\text {sky }}^{-1}$ (see below). This spectrum is comparable to that of the solid line of Fig. 1. Hereafter, only this type of spectra are considered and, consequently, we are concerned with the Residual Effect of Partial Coverage (REPC). Using these spectra, results obtained from squared patches can be compared with results corresponding to the HEALPIX package.

\section{Results}

We first consider simulated maps containing only the CMB signal. The CMB angular power spectrum is used to built up $18^{\circ} \times 18^{\circ}$ maps which have either 256 or 128 nodes per edge. For 256 (128) nodes, the pixel size is $\Delta=4.7^{\prime}$ $\left(\Delta=9.4^{\prime}\right)$ and, in the PLANCK case, the average number of measurements per pixel corresponding to distinct orientations of a given beam is $N_{\mathrm{c}}=3.13\left(N_{\mathrm{c}}=6.26\right)$. Hereafter, the elliptical beam is - implicitly - assumed to be an asymmetric one of the form (1) with $\sigma_{\theta}=6^{\prime}$ and $\sigma_{\phi}=10^{\prime}$, excepting a few cases where other beams are explicitly defined. Moreover, any set of $n 18^{\circ} \times 18^{\circ}$ simulated maps is called a $n$-simulation.

\subsection{Pixelisation and partial coverage}

First of all, the superposition of the REPC and the pixelisation effect is estimated in the absence of any beam. The REPC depends on the coverage and the pixelisation effect depends on the pixel size $\Delta$. Pixelisation is a mathematical discretisation and, consequently, any discretised mathematical formula used in our numerical procedures may induce an effect, which could appear as a deviation of the resulting angular power spectrum with respect to the true one. Discretised mathematical formulae can be involved in the method to extract the spectrum from the maps and even in the simulation procedure. That makes no possible the definition of a pixelisation effect depending only on the pixelisation itself; in each case (signal plus mathematical methods of simulation and analysis), the effect of discretisation (pixelisation) must be estimated. We do that below (for squared patches) and the resulting effect appears to be very systematic, namely, it appears to be almost the same in any simulation. The same occurs when the HEALPIX package is used. Of course, the spectra obtained from our simulations include both the REPC and the pixelisation effect. In Fig. 2, all the spectra are obtained from fifty $18^{\circ} \times 18^{\circ}$ maps (50-simulation) having 256 nodes per edge, namely from a partial sky coverage with $f_{\text {sky }} \simeq 0.39$ and $\Delta=4.7^{\prime}$. The effect of pixelisation becomes dramatic for $\ell>\pi / \Delta$, where $\Delta$ is the pixel size and, consequently, the spectra are only showed for $\ell<\ell_{\max }=\pi / \Delta=2550$. Each spectrum is displayed in two panels (left and right) to make visible some details. In the top panel of Fig. 2, the solid line corresponds to the spectra obtained from a 50-simulation, the dotted line shows the true $C_{\ell}$ coefficients used in the simulations and, finally, the dashed and dotted-dashed lines correspond to $C_{\ell}-\Delta C_{\ell}$ and $C_{\ell}+\Delta C_{\ell}$, where $\Delta C_{\ell}$ is given by Eq. (5). The effect produced by pixelisation is expected to be dominant for large $\ell$-values and this effect appears always mixed with the REPC. For $\ell>1600$ (see top right panel), the solid line is well outside the region limited by $C_{\ell}-\Delta C_{\ell}$ and $C_{\ell}+\Delta C_{\ell}$, which suggests that the effect due to pixelisation is clearly dominant. For $1600>\ell>100$, there is a mixing of the two effects under consideration (which are disentangled below). Finally, the largest values of the REPC should be found for $100<\ell<300$ (see top and middle left panels).

In the middle panel of Fig. 2, the dotted line shows the true $C_{\ell}$ coefficients, whereas each of the other two lines corresponds to the spectrum obtained from a different 50 simulation (these two spectra are denoted $C_{\ell}^{1}$ and $C_{\ell}^{2}$ in this Section). It is noticeable that the deviations of these two lines with respect to the dotted line (true spectrum) are very similar. This fact indicates that - for our signal and numerical procedures -, the addition of the REPC and the pixelisation effect has a dominant systematic part. The average deviations are the quantities $D_{\ell}=C_{\ell}-\left(C_{\ell}^{1}+\right.$ $\left.C_{\ell}^{2}\right) / 2$, and the quantities $\tilde{C}_{\ell}=C_{\ell}^{1}-D_{\ell}$ (hereafter named the corrected spectrum) are given in the bottom panels of Fig. 2 (solid line), where we can verify that quantities $\tilde{C}_{\ell}$ are very similar to the true $C_{\ell}$ coefficients (dotted line) all along the interval $\left(100, l_{\max }\right)$. The dashed and doteddashed lines of this panel have the same meaning that those of the top panel.

In order to give quantitative estimates, some relative deviations $\Delta C_{\ell} / C_{\ell}$ are calculated for appropriate pairs of spectra. These deviations are presented in Fig. 3. The top panel of this figure shows the deviations of the spectra $C_{\ell}^{1}$ and $C_{\ell}^{2}$ of Fig. 2 with respect to the true $C_{\ell}$ coefficients. In both cases, quantities $\Delta C_{\ell} / C_{\ell}$ are similar, which suggests a systematic effect (deviation with respect to the true spectrum). Furthermore, the maxima and minima of $\Delta C_{\ell} / C_{\ell}$ seems to be associate to the minima and maxima in $\ell(\ell+1) C_{\ell}$, respectively. Finally, the bottom panel of Fig. 3 shows the relative deviations between $\tilde{C}_{\ell}$ and $C_{\ell}$, which are hereafter called residual deviations. The largest residual deviations are now of a few percent for any $\ell$, and the peaks of this curve do not correspond to maxima and minima in the angular power spectrum. The systematic deviations have been ruled out, and the residual deviations depend on the pair of simulations under consideration.

In order to separate the pixelisation effect and the REPC, the above study has been repeated in two cases: (i) the previous pixelisation is maintained, whereas a 500simulation is used; hence, the new coverage is ten times larger than the previous one. For the sake of briefness, no figures are presented. We only describe our main conclusions: (1) The systematic deviations has kept almost unaltered and, (2) after subtracting these deviations, the residual ones range in the interval $(-0.07,0.07)$; namely, they are a factor $10^{-1 / 2}$ smaller than those of the bottom panel of Fig. 3. These facts suggest that the systematic effect is due to pixelisation, not to partial coverage, whereas after subtraction, the resulting effect is due to 

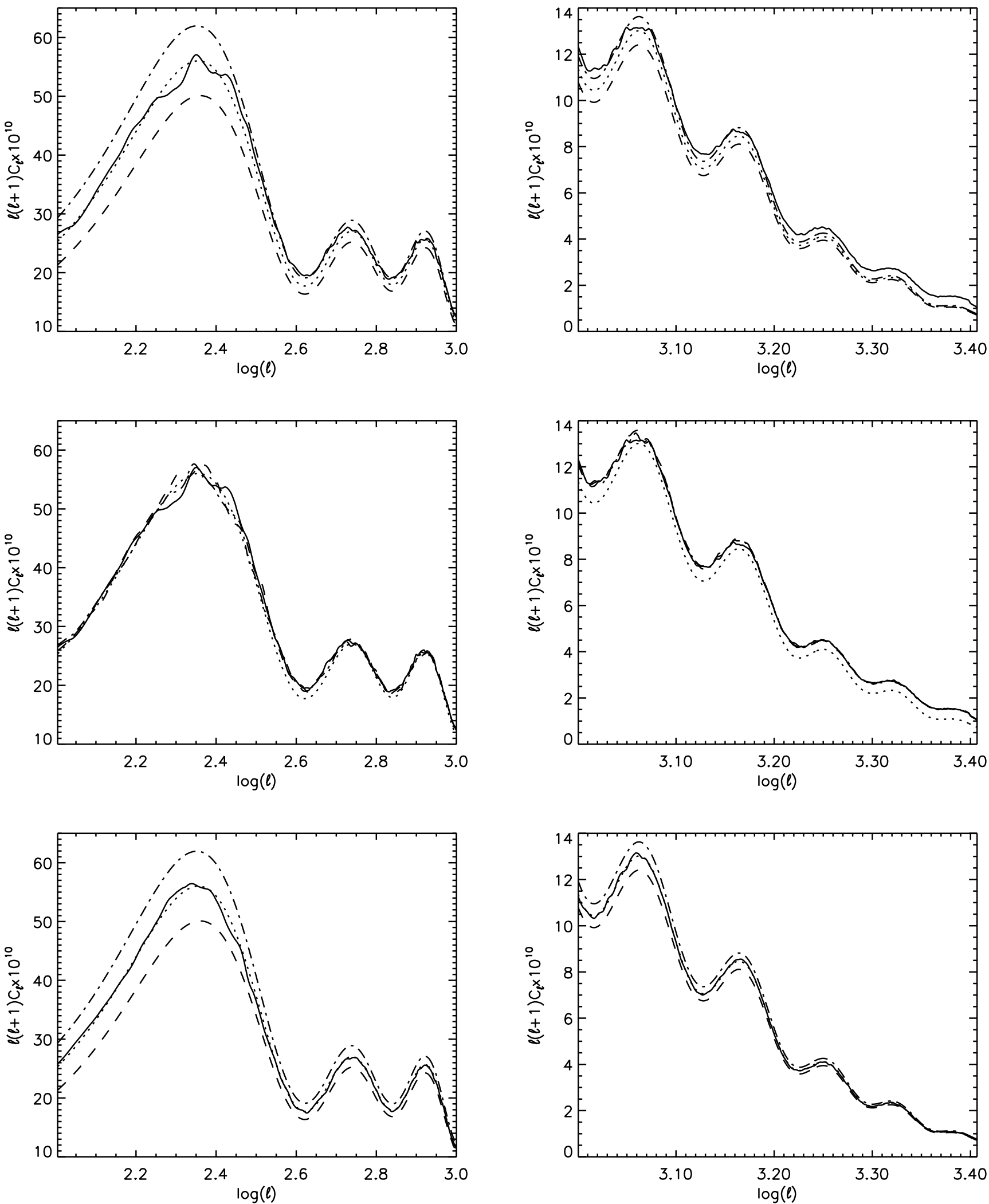

Fig. 2. Left (right) top panel shows four spectra for $\ell<1000(\ell>1000)$ : dotted lines display the theoretical spectrum used to simulate $18^{\circ} \times 18^{\circ}$ maps with 256 nodes per edge and no beam. Solid line is the spectrum obtained from 50 simulated maps (50-simulation). Dashed (dotted-dashed) lines corresponds to $C_{\ell}-\Delta C_{\ell}\left(C_{\ell}+\Delta C_{\ell}\right)$, where $\Delta C_{\ell}$ is given by Eq. (5). Middle panels show three spectra: dotted line is the theoretical spectrum and solid and dashed lines are the spectra obtained from two independent 50-simulations. In bottom panels, dotted, dashed and dotted-dashed lines show the same spectra as in top panel, but solid line is the spectrum obtained from simulations after subtracting (see text) the systematic effect due to pixelisation. 

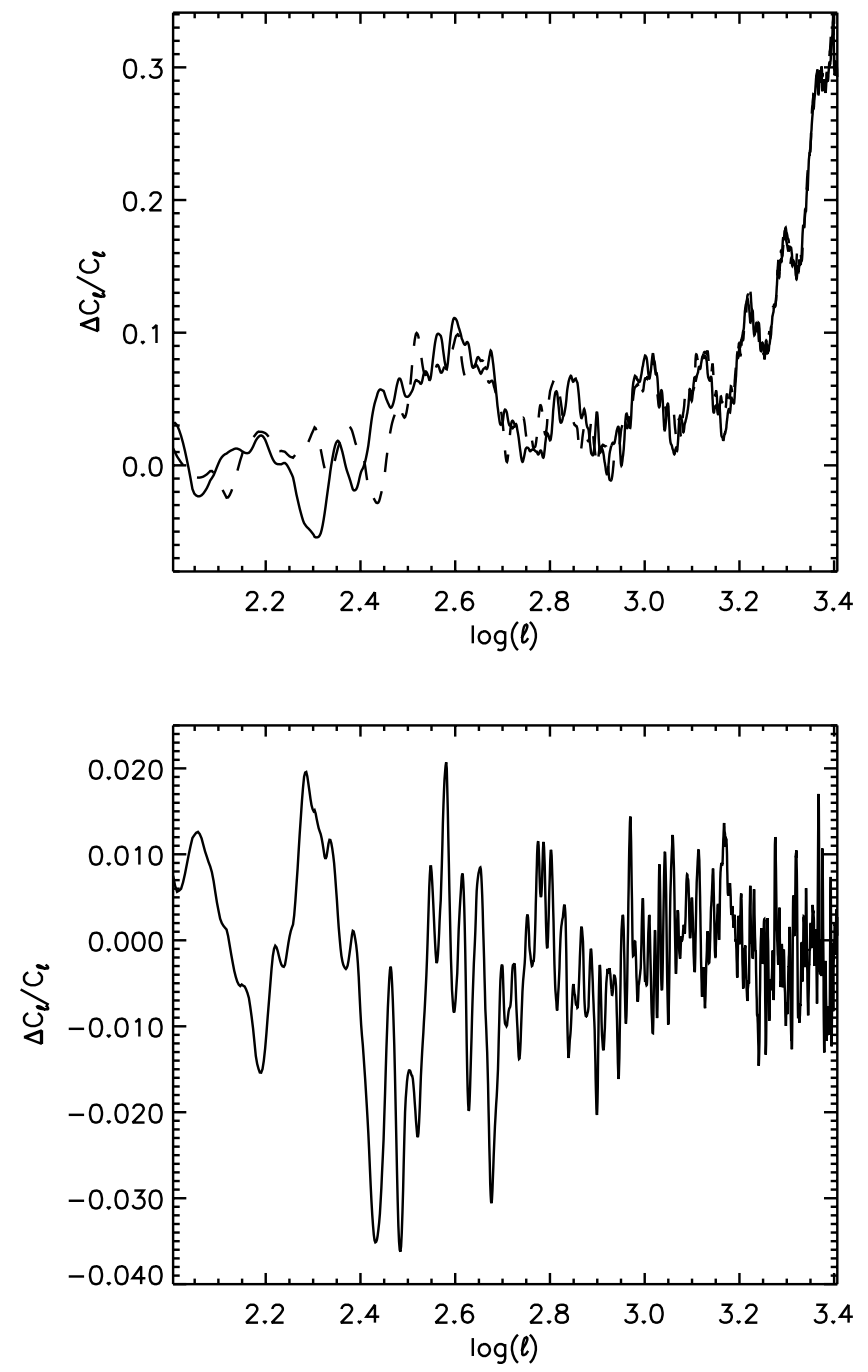

Fig. 3. Solid and dashed lines of the top panel give the relative deviations between the theoretical spectrum and those corresponding to the solid and dashed lines of the middle panels of Fig. 2. Bottom panel gives the residual deviations defined in the text.

partial coverage (it is the REPC proportional to $f_{\text {sky }}^{-1 / 2}$ ). In the case (ii) a 50-simulation is used (the initial coverage), and a new pixelisation with 128 nodes per edge is assumed. In such a case, the spectrum can be only obtained for $\ell<\ell_{\max }=\pi / \Delta=1275$ and, for these $\ell$ values we observe that: (a) a systematic effect appears again, (b) the maxima and minima of $\Delta C_{\ell} / C_{\ell}$ are again associated to the minima and maxima in $\ell(\ell+1) C_{\ell}$, respectively, and (c) after subtraction of the systematic deviations, the residual deviations are very similar to those of the bottom panel of Fig. 3, as it should occur if such deviations are due to the unaltered partial coverage.

From the above considerations it follows that - after estimating and eliminating systematic effects due to pixelisation - the spectrum extracted from fifty $18^{\circ} \times 18^{\circ}$ maps is very accurate for $100<\ell<\ell_{\max }$.

The above study about the REPC and the pixelisation effect has been repeated using the HEALPIX
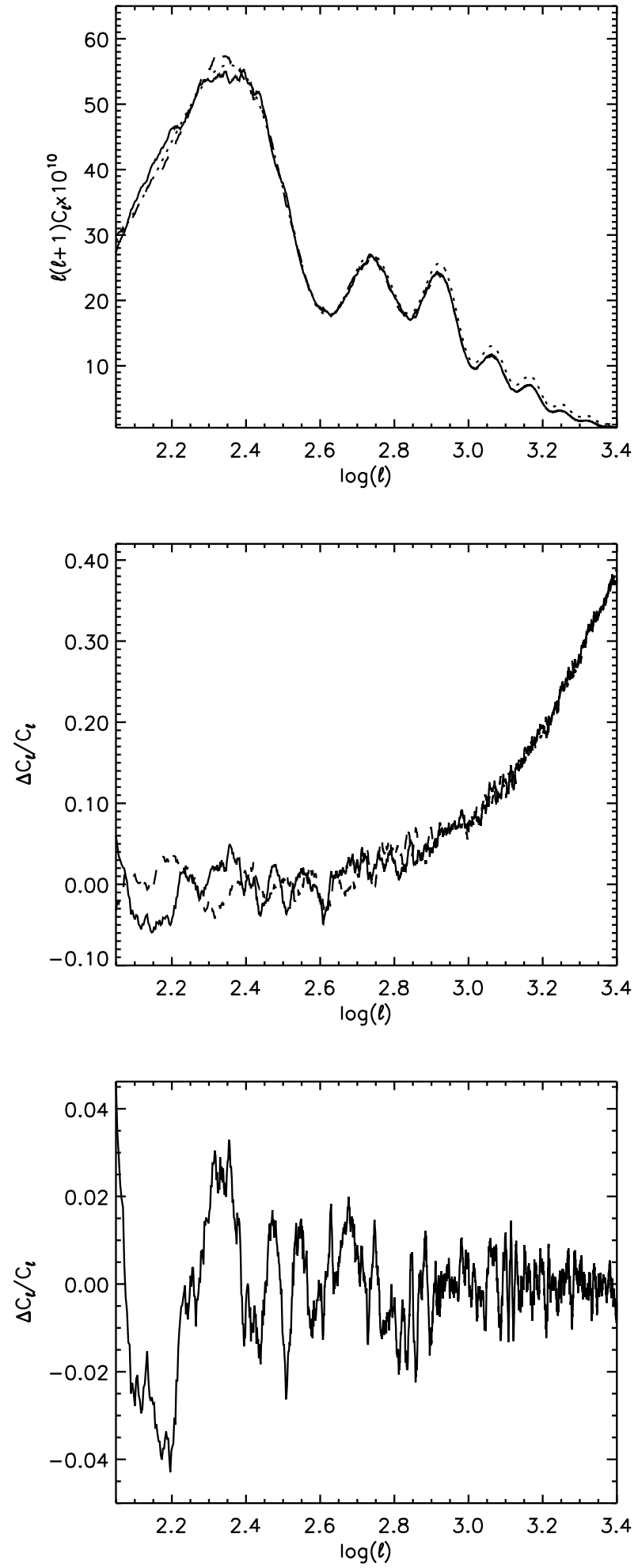

Fig. 4. Top panel is the same as the middle panels of Fig. 2, and middle (bottom) panel is the same as the top (bottom) panel of Fig. 3. All the simulations are performed using HEALPIX with $\Delta=3.435$.

package (see Sect. 3). Two polar regions have been considered in each simulation $\left(f_{\text {sky }} \simeq 0.39\right)$. The pixel size is $\Delta \simeq 3.435$; hence, we work with the same total sky coverage as in the case of squared patches, but with a different 
$\Delta$ value compatible with HEALPIX. Results from two independent simulations are presented in Fig. 4. In the top panel, the solid and dashed lines show the spectra obtained from these two simulations. These lines are almost indistinguishable and, consequently, they deviate almost the same with respect to the dotted line (true spectrum). The relative deviations (residual deviations) defined above are given in the middle (bottom) panel. Residual deviations are similar to those obtained using patches, which is not surprising because the same sky coverage $\left(f_{\text {sky }} \simeq 0.39\right)$ has been assumed in both cases. Using simulations with 20 polar regions (sky coverage enhanced by a factor ten), we have verified that the residual deviations reduce by a factor $\sim 10^{1 / 2}$. We have also used two polar regions with $\left(f_{\text {sky }} \simeq 0.39\right)$ and a greater pixel size $\Delta=6.87$ to get comparable residual deviations. In short, using HEALPIX and polar regions (where the pixel shapes are more irregular), the same qualitative results as in the case of squared patches have been obtained: (1) the pixelisation effect appears to be very systematic, (2) the residual deviations correspond to the REPC and, (3) the pixelisation effect and the REPC (residual deviations) can be easily disentangled. In next sections, only methods based on squared patches are used.

\subsection{Asymmetric non-rotating beam}

The effect due to beam asymmetry is first estimated in the absence of rotation. Maps are smoothed with both the elliptical beam (which is oriented in the same way everywhere) and its circular associated one (see Sect. 2). These beams lead to different averages at each node and, consequently, they produce different alterations of the true angular power spectrum. Results are presented in Fig. 5, whose top (middle) panel corresponds to maps with 256 (128) nodes per edge. In these panels, the spectra are only shown in the $\ell$ interval where the differences among them are more relevant: $\ell>1000(\ell>360)$ for 256 nodes (128 nodes). The solid line gives the spectrum after smoothing with the spherical associated beam (which is denoted $C_{\ell}$ in this section). The dotted and dashed lines give the spectra obtained from two independent 50simulations after smoothing with the nonrotating asymmetric beam (these spectra are denoted $C_{\ell}^{1}$ and $C_{\ell}^{2}$ along this section). These two lines are very similar and, consequently, they deviate almost the same with respect to the solid line. This means that the effect due to asymmetry (without rotation) is a very systematic one. The relative deviations between $C_{\ell}$ and $C_{\ell}^{1}$ and between $C_{\ell}$ and $C_{\ell}^{2}$ measure the effect of beam asymmetry without rotation. These deviations are displayed in the bottom panel of Fig. 5, where curves I and II (III and IV) correspond to the $C_{\ell}^{1}$ and $C_{\ell}^{2}$ spectra displayed in the top (middle) panel. For the $256 \times 256$ pixelisation (top panel), the deviations are smaller than $\sim 8 \%$ all along the interval $\left(100, \ell_{\max }\right)$, whereas for the $128 \times 128$ pixelisation (middle panel), these deviations are greater than $\sim 8 \%$ for $\ell>700$, reaching
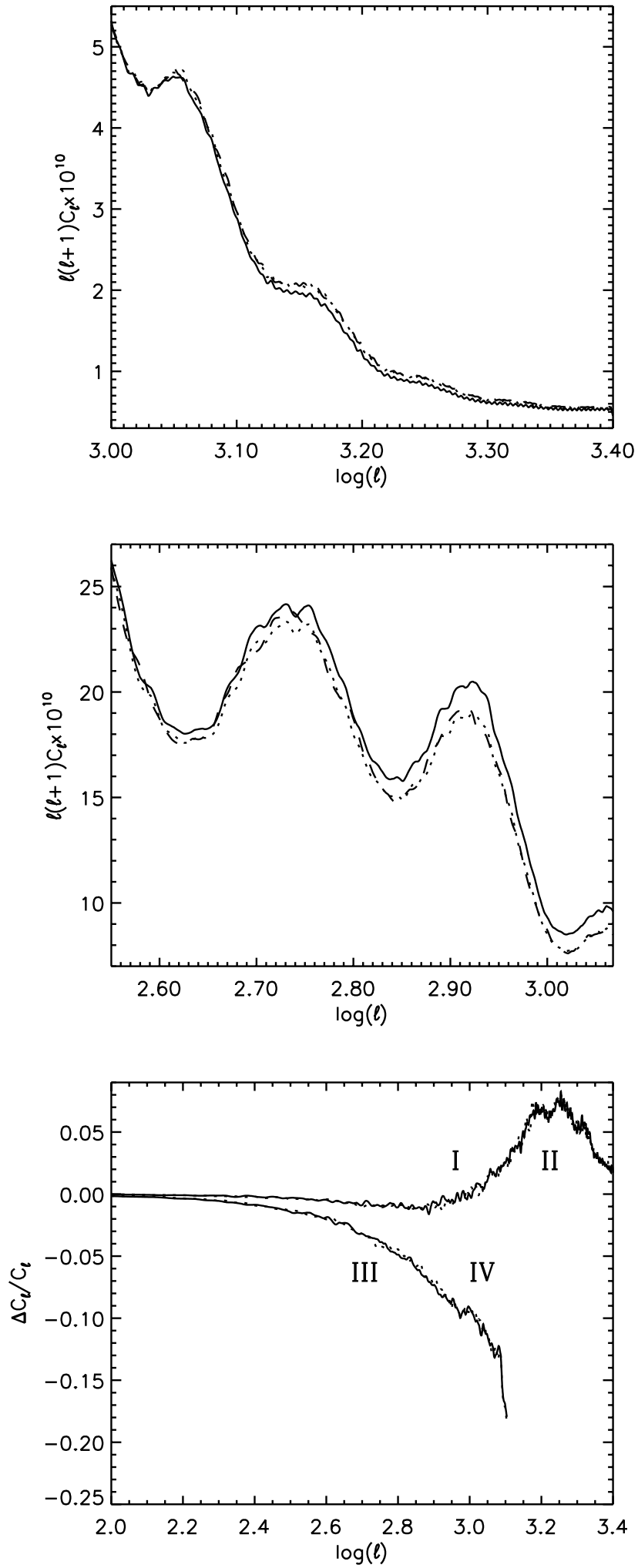

Fig. 5. Top (middle) panel shows three spectra obtained from 50 -simulations with $256 \times 256(128 \times 128)$ nodes per edge. In both panels, solid line is the spectrum obtained from a 50 simulation after smoothing with the circular associated beam and the other lines correspond to the same and to another independent 50-simulation after smoothing with the asymmetric non-rotating beam. Lines I and II (III and IV) of the bottom panel give the relative deviations between the solid line and each of the other two lines in the top (middle) panel. 
values close to $\sim 20 \%$ near $\ell=\ell_{\max }$. There is a dependence on the pixelisation in the sense that, the smaller the pixel size the smaller the relative deviations (in the part of the spectrum common to both pixelisations). Curves I and II are very similar, and the same occurs with curves III and IV, which means that the effect under consideration is actually systematic. If the systematic effect is subtracted as in Sect. 4.1; namely, if the quantities $D_{\ell}$, the corrected spectrum $\tilde{C}_{\ell}$, and the residual deviations are calculated, these last deviations are very similar to those showed in the bottom panel of Fig. 3, (associated to the REPC in Sect. 4.1); they are oscillations having amplitudes of a few per cent. Indeed, when 500-simulations are considered, the amplitude of the residual deviations appear divided by $10^{1 / 2}$ as it is expected in the case of any deviation due to partial coverage (see Eq. (5)). Finally, the deviations between $C_{\ell}$ and $C_{\ell}^{1}$ (or $C_{\ell}^{2}$ ) decrease as the assumed level of beam asymmetry does.

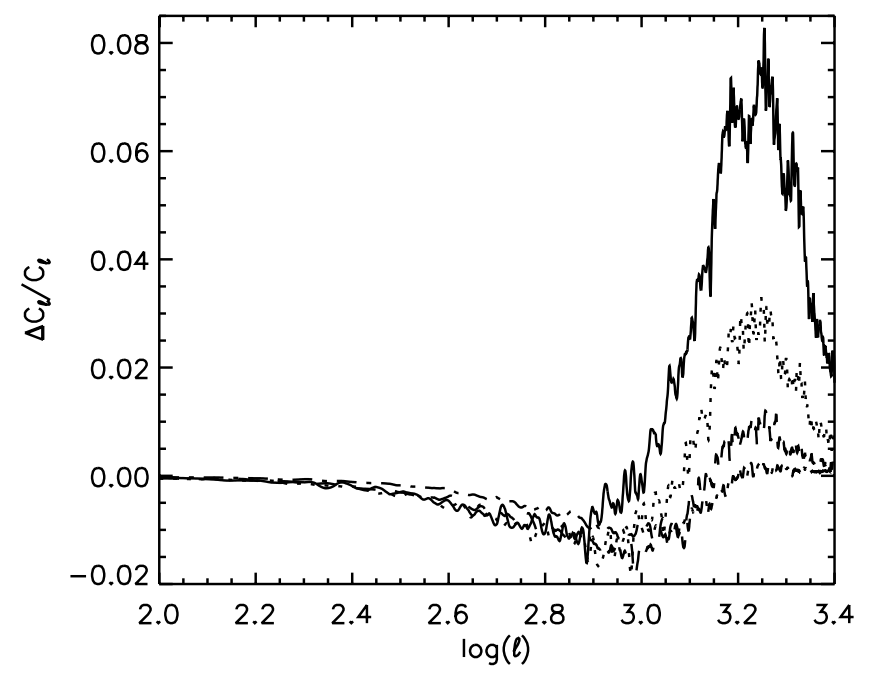

Fig. 6. Relative deviations between the spectrum obtained from a 50 -simulation - with $256 \times 256$ nodes per edge - after smoothing with the circular associated beam, and that obtained from the same 50-simulation after smoothing with the asymmetric non-rotating beam. From top to bottom, curves correspond to different elliptical beams, in which the ratio $\sigma_{\phi}: \sigma_{\theta}$ is $10: 6,10: 7,10: 8$, and 10:9.

In Fig. 6, these deviations are shown for the $256 \times 256$ pixelisation and different elliptical beams. All these beams have $\sigma_{\phi}=10^{\prime}$, whereas $\sigma_{\theta}$ takes on the values $6^{\prime}, 7^{\prime}, 8^{\prime}$, and $9^{\prime}$. The decreasing has been also tested for the $128 \times$ 128 pixelisation but no figure is presented by the sake of briefness.

\subsection{Asymmetric rotating beam}

Various types of rotation strategies - of the asymmetric beam - are now introduced with the essential aim of estimating the rotation effects on the resulting angular power spectra. Of course, these effects are deviations with respect to the spectrum obtained - for the same asymmetric beam - in the absence of rotation $\left(C_{\ell}\right.$ quantities in this section). Two kinds of rotation strategies are considered: in the first one, the beam orientation in each pixel is that corresponding to a beam that does not rotate around its centre, but it describes a big circle on the sky with and aperture angle of $85^{\circ}$. This first case mimics PLANCK observational strategy and it is hereafter named Systematic Rotation (SR); in the second strategy, the angle defining the beam orientation in each pixel is assumed to be a random uniformly distributed variable; hereafter, Random Rotation (RR). In each case, the rotating beam smoothes fifty $18^{\circ} \times 18^{\circ}$ regions of the sky which are distributed without overlapping and with random orientations. In this section, $C_{\ell}^{1}$ and $C_{\ell}^{2}$ stand for two spectra obtained from independent 50-simulations after a smoothing based on one of the above rotation strategies (in each case, this strategy must be explicitly chosen). Each of the fifty $18^{\circ} \times 18^{\circ}$ regions is smoothed only one time with the asymmetric rotating beam. That is a good procedure for experiments where the beam orientation varies from pixel to pixel (according to SR and RR strategies), but measurements are always performed with the same orientation in each pixel. Results are displayed in Fig. 7, where left (right) panel corresponds to SR (RR) strategy. Left and right panels have the same structure, which is identical to that of Fig. 5; hence, on account of the definitions of $C_{\ell}, C_{\ell}^{1}$ and $C_{\ell}^{2}$ given in this section, the meaning of each line (and mark type) is known and we can begin with the necessary discussion. Top panels $\left(\Delta=4.7^{\prime}\right)$ show very different behaviours for $\ell>1600$; while the RR produces an important deviation of the dotted and dashed lines (two independent simulations) with respect to the solid one (no rotation) for $\ell>1600$, the SR does not produces such a deviation. The deviations are described by quantities $\Delta C_{\ell} / C_{\ell}$, which are displayed in curves I and II (bottom panel). Although these curves correspond to two independent 50simulations, they are very similar, which means that the effect under consideration is very systematic; in fact, the residual deviations (after subtraction of the systematic effect) are of a few percent as in Sect. 4.1 (see the bottom panel of Fig. 3).

Middle panels show that, for $\Delta=9.4^{\prime}$, SR and RR produce similar effects in all the interval $\left(100, \ell_{\max }\right)$, where $\ell_{\max }=1275$. For these $\ell$ values, top panels also show similar effects for SR and RR. Relative errors are given in curves III and IV of the bottom panels. The effect is again a systematic one and the residual deviations has the same characteristics as in the case $\Delta=4.7^{\prime}$ (top panels), which is easily understood taken into account that top and middle panels correspond to the same partial coverage.

Finally, a pair of numerical experiments whose results are not displayed in figures: (i) we have increased the coverage using 500-simulations and the main effect is that the new spectra are very similar to those of Fig. 7, but they have much smaller high frequency oscillations (all the curves are much more smooth), which is associated to the decreasing of the residual deviations (the same behaviour have been already found in Sects. 4.1 and 4.2), and (ii) 

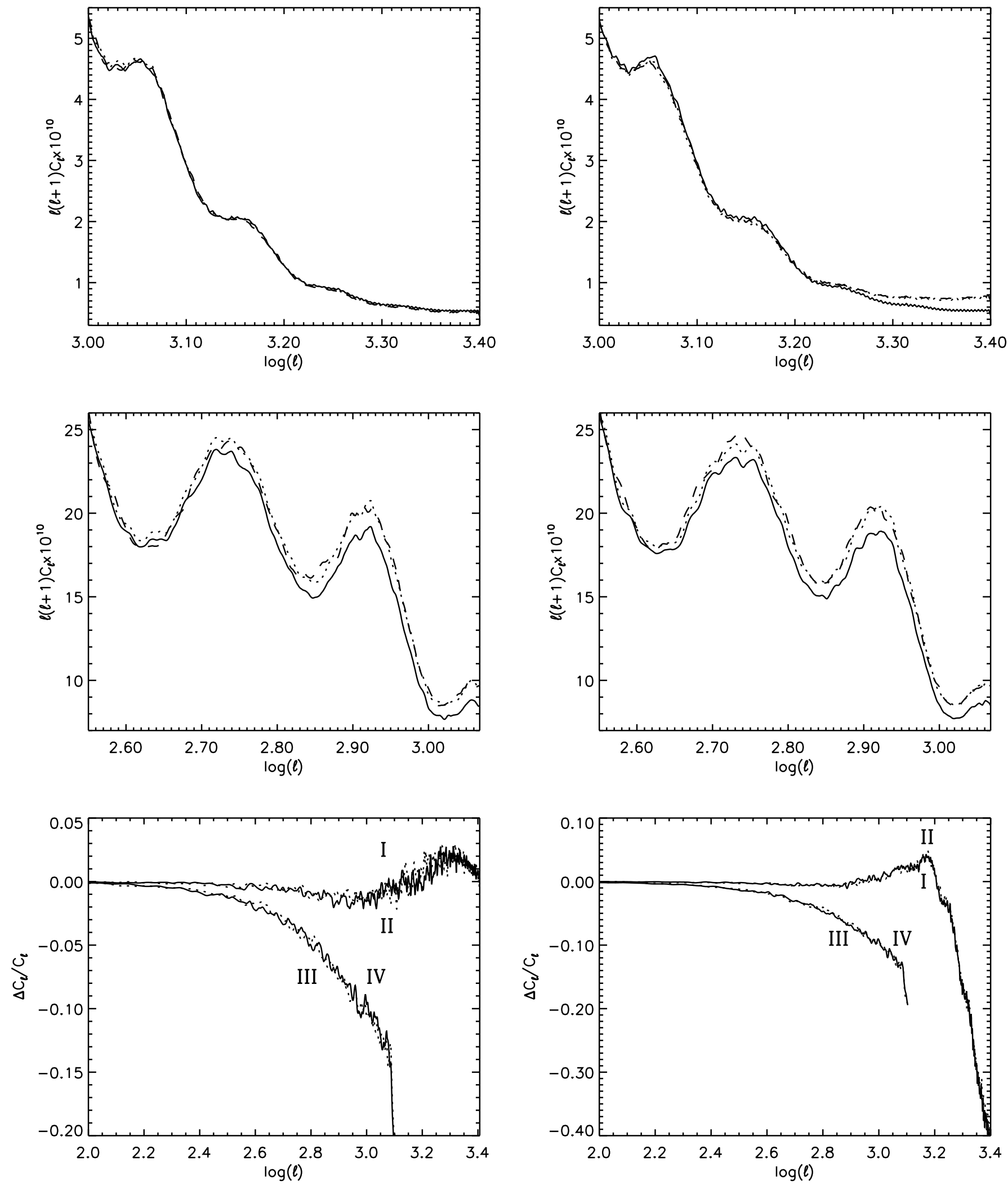

Fig. 7. Top left panel shows three spectra for $\ell>1000$ : solid line is the spectrum obtained from a 50-simulation with $256 \times 256$ nodes per edge after smoothing with the asymmetric non-rotating beam. Dashed and dotted lines are the spectra obtained from the same simulation and another independent one, after smoothing with the same asymmetric beam following the SR strategy defined in text. Top right panel is the same as the top left one for the RR strategy. Middle panels are as the top ones but simulations have $128 \times 128$ nodes per edge. Lines I and II (III and IV) of the bottom panel gives the relative deviations between the solid line and each of the other two lines in the top (middle) panel. 
in the second experiment, each of the fifty $18^{\circ} \times 18^{\circ}$ regions have been smoothed $N_{\mathrm{c}}$ times. Now, the orientation varies from pixel to pixel in each smoothing (according to $\mathrm{SR}$ and RR strategies) and, furthermore, the orientation changes inside a given pixel from smoothing to smoothing. Thus, we account for the fact that, in the framework of some experiments (PLANCK mission), each pixel is observed $N_{\mathrm{c}}$ times with different orientations. For the SR strategy (which is similar to that of PLANCK), the minimum $N_{\mathrm{c}}$ value is taken to be equal to the entire part of the number $N_{\mathrm{c}}$ estimated in Sect. 1 ; hence, we take $N_{\mathrm{c}}=3$ for $\Delta=4.7^{\prime}$ and $N_{\mathrm{c}}=6$ for $\Delta=9.4^{\prime}$; we also take the values $N_{\mathrm{c}}=30$ (for $\Delta=4.7^{\prime}$ ) and $N_{\mathrm{c}}=60$ (for $\Delta=9.4^{\prime}$ ) in order to consider the existence of multiple detectors in current or future experiments. In the case of the RR strategy, we take the same $N_{\mathrm{c}}$-values. Even for the greatest $N_{\mathrm{c}}$ values, no appreciable differences are observed with respect to the case $N_{\mathrm{c}}=1$. The same residual deviations and high frequency oscillations appear in all the cases; this result is not surprising taking into account that: (1) the partial coverage is that of a 50-simulation whatever the $N_{\mathrm{c}}$ value may be and, consequently, the residual deviations do not decrease as we smooth the same 50-simulation various times and, (2) the effect produced by beam rotation is a systematic one which is obtained very accurately from the first smoothing of the 50-simulation. This means that, if a second smoothing is performed, the resulting spectrum must be very similar to the first one, and the average spectrum should be also almost identical to that of each smoothing (with no cancellation of the contribution due to the REPC); this means that, if the coverage is large (very systematic deviations), the $N_{\mathrm{c}}$ value is irrelevant.

\subsection{Including galactic foregrounds}

In previous sections, the effects produced by beam asymmetry and rotation have been described in detail for 50simulations of the CMB signal. These effects appear to be rather small and very systematic. Is that due to the homogeneous and isotropic statistical character of the CMB temperature distribution?

What happens with other types of distributions? The CMB is contaminated by galactic and extragalactic foregrounds which are not homogeneous and isotropic statistical fields and, consequently, the following question arises: what can we say about asymmetry and rotation effects in the presence of the most important galactic foregrounds? Are these effects very different from those estimated in previous sections?

The galaxy produces an unique temperature distribution on the sky at a given frequency, which must be known (from observations) before possible subtraction. We are interested in a frequency channel involving small galactic contamination. The chosen frequency is $100 \mathrm{GHz}$. We have used synchrotron and dust maps of the full sky, which were taken from ESA (the maps and their technical description are freely available at astro.estec.esa.nl). These maps
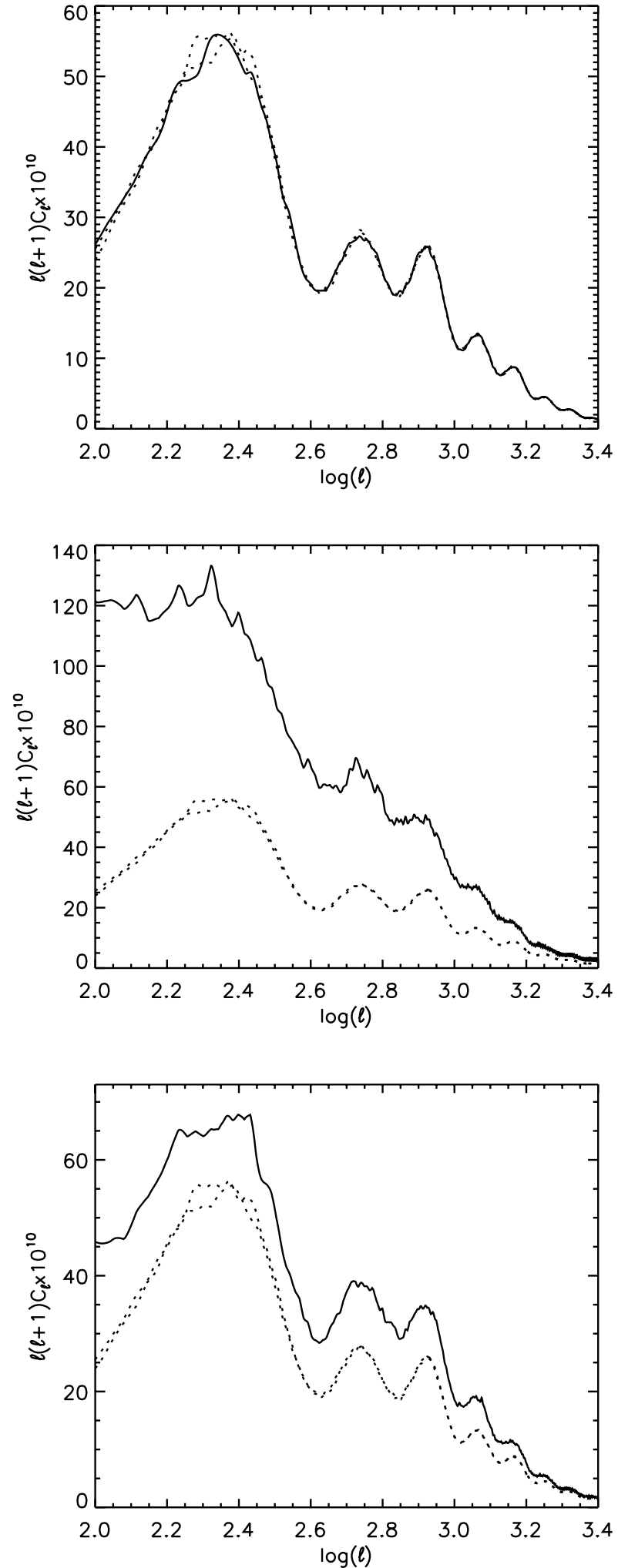

Fig. 8. In all panels, solid line shows the spectrum of the CMB plus synchrotron and dust radiation from a part of the Milky Way, and dotted and dashed lines are the spectra extracted from two independent 50-realizations of pure CMB. No beam has been used at all. All the spectra have been found from fifty $18^{\circ} \times 18^{\circ}$ regions of the sky with $256 \times 256$ nodes per edge. Top, middle and bottom panels corresponds to the regions of the sky G4-G5, G1-G2, and G3-G4, respectively. These regions are defined in the text. 

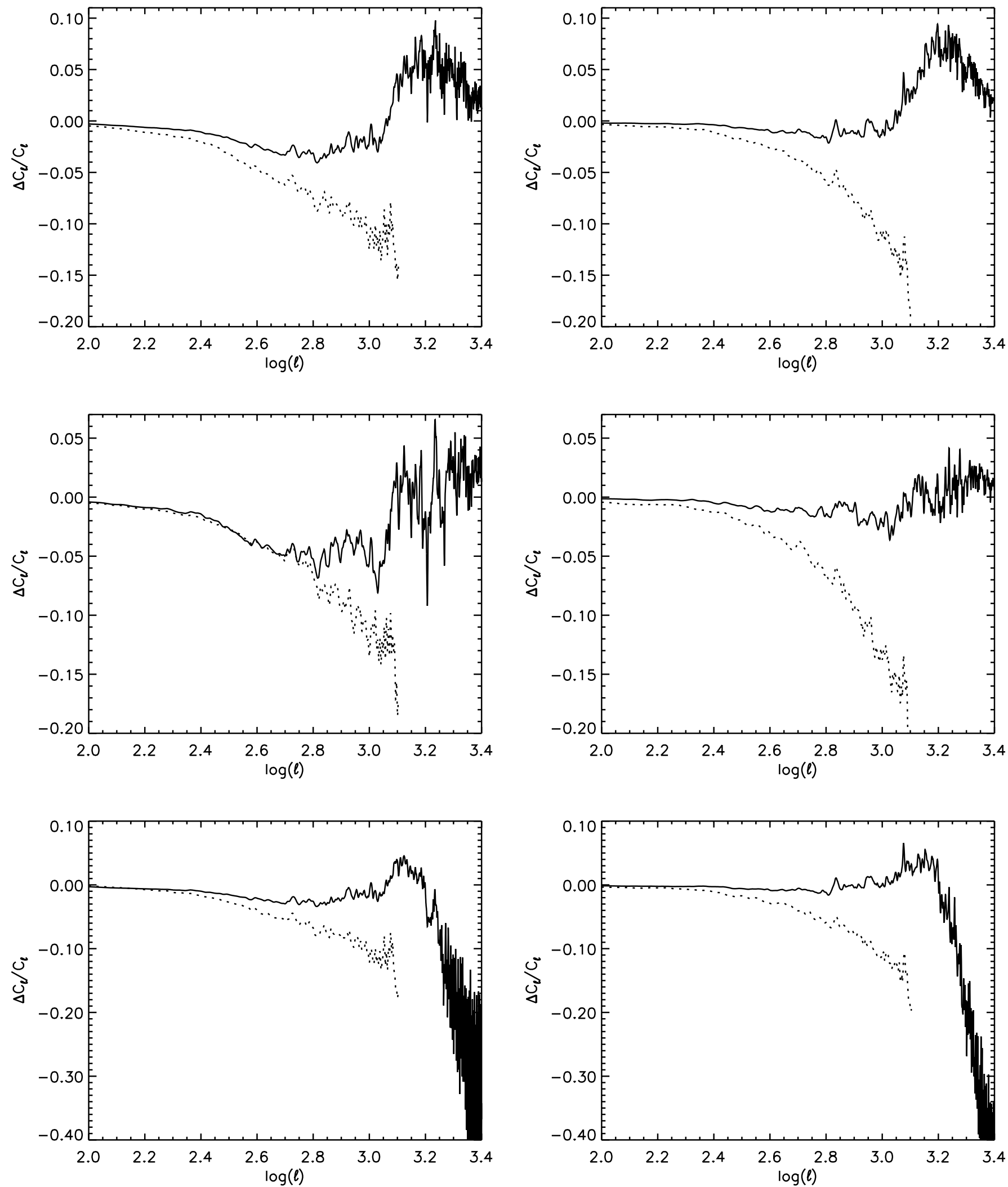

Fig. 9. Left (right) panels correspond to 50f-simulations - see text - containing synchrotron and dust radiations from regions G1 and G2 (G3 and G4) defined in the text. Top panels show deviations between the spectra extracted from a 50f-simulation in two cases: after smoothing with the circular associated beam, and after using the non-rotating asymmetric beam. Middle (bottom) panels also show deviations, but the compared spectra are obtained after smoothing with the non-rotating asymmetric beam and with the rotating one in the case of SR (RR). Solid (dotted) lines correspond to $256 \times 256(128 \times 128)$ nodes per edge. 
were designed by G. Giardino and P. Fosalba and they have HEALPIX structure and a pixel size $\Delta=1.7^{\prime}$. Using these maps and an appropriate smoothing, we have built up one hundred and fifty $18^{\circ} \times 18^{\circ}$ maps with $\Delta=4.7^{\prime}$ and the same number of maps with $\Delta=9.4^{\prime}$.

50-Simulations of the CMB signal are obtained as in previous Sections, and groups of fifty $18^{\circ} \times 18^{\circ}$ maps with the galactic foregrounds are appropriately selected (see below); then, each of the $18^{\circ} \times 18^{\circ} \mathrm{CMB}$ maps is superimposed to one of the $18^{\circ} \times 18^{\circ}$ foreground maps to get a 50f-simulation (which includes the CMB signal plus synchrotron and dust from the Milky Way). Then, the study of previous sections is performed on the resulting 50f-simulation.

In order to get $18^{\circ} \times 18^{\circ}$ maps with the foregrounds, a cube is inscribed in the celestial sphere in such a way that the centres of the faces are distributed as follows: (i) centres 1 and 2 point towards the centre of the Milky Way and its opposite direction, centres 3 and 4 point toward two opposite directions which are contained in the galactic plane and are orthogonal to the direction joining centres 1 and 2 and, (iii) centres 5 and 6 point towards the two galactic poles. Each of the six faces is divided in 25 regions, in such a way that after projection on the sphere, our 150 maps are almost squared and have similar areas and a small overlapping. The 25 maps corresponding to the face with centre at $i$ are hereafter named the 25-group $G_{i}$.

The first 50f-simulation is built up using the 25 -groups $G_{5}$ and $G_{6}$ localized around the galactic poles. The solid line in the top panel of Fig. 8 gives the spectrum corresponding to this 50f-simulation, which can be compared with the two spectra showed in the pointed and dashed lines, which correspond to two independent 50-simulations without galactic foregrounds. No beam has been considered at all. The small differences among these curves appear as a result of the REPC, and the galactic contribution contained in the continuous line is hidden by this dominant effect.

The second (third) 50f-simulation includes the 25groups $G 1$ and $G 2$ ( $G 3$ and $G 4$ ); hence, the second (third) 50f-simulation includes the Milky Way centre (a part of the galactic disk). The effect of the galactic foregrounds is not negligible. That can be seen in the middle (second 50f-simulation) and bottom (third 50f-simulation) panels of Fig. 8, where the spectra containing the galactic foregrounds (solid line) are very different from the spectra corresponding to 50-simulations of pure CMB (dotted and dashed lines). No beam has been used.

The same asymmetric beam and rotational strategies as in previous sections have been used to smooth each 50f-simulation. Results are presented in Fig. 9. Left (right) panels corresponds to the second (third) 50f-simulation. In all the panels, the solid (dotted) line shows results from $256 \times 256(128 \times 128)$ simulations. All the panels give relative deviations between two spectra, which are obtained after smoothing with: (i) the non rotating beam and the circular associated one in the top panels, (ii) the non rotating beam and the rotating one with SR strategy in the middle panel and, (iii) the non rotating beam and the rotating one with RR strategy in the bottom panels. The top panel measures the effect of beam asymmetry without rotation, this panel should be compared with the bottom panel of Fig. 5, which shows the same effect in the absence of galactic foregrounds. The middle (bottom) panel gives the effect due to SR (RR) strategy and, consequently, this panel must be compared with the left (right) bottom panel of Fig. 7. The comparisons show some appreciable differences produced by the presence of significant galactic foregrounds (see Fig. 8). In Fig. 9, the high frequency oscillations are most important that in the figures used for comparison; particularly, for large $\ell$ values. That could be due to the presence of oscillations in the angular power spectrum of the galactic foregrounds (see Fig. 8 for the same $\ell$ values). For small and intermediate $\ell$ values, the foreground contributions to the angular power spectrum are significant (see Fig. 8) and, consequently, there are also appreciable differences between the $\Delta C_{\ell} / C_{\ell}$ deviations displayed in Fig. 9 and those of the figures used for comparison (without foregrounds). In spite of the fact that the assumed foregrounds are not homogeneous and isotropic statistical fields, the mentioned differences are not very large. They are more important in the case of the left panels, in which, directions pointing close to the Milky Way centre have been considered. For the first 50fsimulation (galactic poles) the $\Delta C_{\ell} / C_{\ell}$ deviations are not presented in figures because no appreciable differences appear in this case with respect to 50-simulations without foregrounds.

\section{Discussion}

In the absence of beam, the pixelisation effect and the REPC have been disentangled to conclude that pixelisation produces very systematic deviations with respect to the true angular power spectrum. This conclusion has been obtained using two very different methods for simulations and data analysis (see Sect. 3)

We have studied the deviations in the angular power spectrum produced by the rotation of an asymmetric beam. Two rotation strategies have been considered. One of them (SR) is similar to that of future experiments as PLANCK. The second strategy (RR) is very different from SR, and it has been introduced for comparisons. Maps with and without the dust and synchrotron radiations from the Milky Way (at $100 \mathrm{GHz}$ ) have been considered. In Sect. 4, the rotation effects corresponding to different cases have been described and compared, now let us present some general comments.

If radiation from the galaxy is not considered, the most important conclusion is that rotation effects are very systematic for any rotation strategy and $f_{\text {sky }}=0.39$. They are so systematic that we can subtract the deviations appeared in a 50-simulation, from the spectrum of another one, to recover very well the spectrum corresponding to the nonrotating beam (except for small deviations which 
seem to be essentially due to the REPC). Furthermore, the resulting effects depend on the rotation strategy, in particular, for large $\ell$ values and, consequently, they must be estimated - using simulations - in each particular case.

Radiation from the galaxy - which can be seen as a non homogeneous an non isotropic statistical field - contributes significantly to the observable signal, except in the case of the polar galactic regions (G5-G6). In the G1-G2 and G3-G4 cases, the effect of beam rotation is significantly, but not dramatically, different from that obtained in the absence of foregrounds.

After the deviations in the angular power spectrum due to beam asymmetry and rotation have been estimated and characterized (the main goal of this paper) and, after proving that beam effects are very systematic, some practical applications can be easily outlined.

Take the CMB power spectrum corresponding to a certain theoretical model of structure formation in a given universe, take also a model for the foregrounds, a pixelisation, the asymmetric beam for a given frequency, and the rotation strategy of an experiment with a large enough coverage (i.e. PLANCK), and then, use a simulation -as the 50-simulations of this paper or similar - to find the spectrum $C_{\ell}^{\mathrm{CMB}}$ after smoothing with the asymmetric rotating beam. Repeat the simulation a large enough number of times and verify that the resulting $C_{\ell}^{\mathrm{CMB}}$ spectra are similar in all cases (systematic character). Finally, use the deviations among the resulting spectra to assign an error bar to $\left\langle C_{\ell}^{\mathrm{CMB}}\right\rangle$. Use these data - obtained from simulations - to answer the following question: is the theoretical model under consideration compatible with the observational data from the experiment? In order to find the answer, the observational data could be analyzed as follows: (i) Eliminate a part of the instrumental noise using an appropriate method (wavelets, Fourier transform, and so on), (ii) Separate components (CMB, synchrotron from our galaxy, and so on) taking into account the frequency dependences, but keeping beam smoothings unaltered (usually, the beams are eliminated at this stage under simplifying assumptions and without considering rotation), (iii) use the map of the CMB component which has already been separated from foregrounds - to extract the experimental spectra, $C_{\ell}^{\mathrm{CMB}}(\exp )$, and finally (iv) compare $C_{\ell}^{\mathrm{CMB}}$ with $C_{\ell}^{\mathrm{CMB}}(\exp )$ and study if these spectra can be identified taking into account the error bars. If they can, the theoretical model is compatible with observations. Note that - at the last step of the process we compare a simulated spectrum with an observational one, and note also that both spectra are obtained from maps which have been smoothed with the same rotating asymmetric beam; hence, the proposed method for data analysis includes beam rotation, treating it (after verification) as the source of a very systematic effect. Of course, this method has been only outlined, and much more work would be necessary before implementation.

Acknowledgements. Part of this work was supported by the Spanish MCyT (project AYA2000-2045). Some calculations were carried out on a SGI Origin 2000s at the Centro de Informática de la Universidad de Valencia. We wish to thank an anonymous referee for constructive comments.

\section{References}

Arnau, J. V., \& Sáez, D. 2000, New Astron., 5, 121

Bond, J. R., \& Efstathiou, G. 1987, MNRAS, 226, 655

Burigana, C., et al. 1998, A\&AS, 130, 551

Hanany, S., Jaffe, A. H., \& Scannapieco, E. 1998, MNRAS, 299, 653

Knox, K. 1995, Phys. Rev. D, 52, 4307

Sáez, D., \& Arnau, J. V. 1997, ApJ, 476, 1

Sáez, D., Holtmann, E., \& Smoot, G. F. 1996, ApJ, 473, 1

Scott, D., Srednicki, M., \& White, M. 1994, ApJ, 421, L5

Wu, J. H. P., et al. 2001, ApJS, 1, 132 